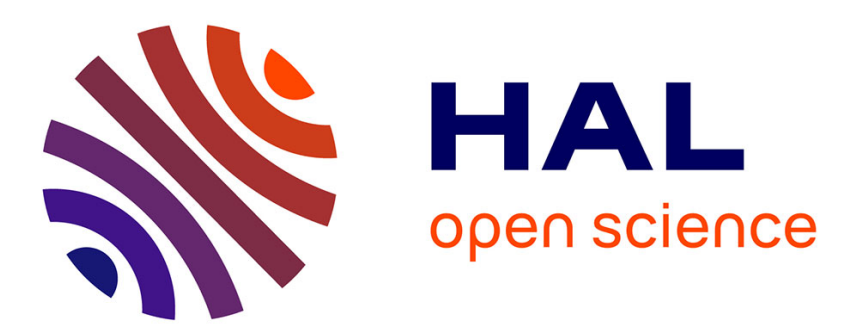

\title{
Statistiques de la population et politique des catégories aux États-Unis au xixe siècle. Théories raciales et questions de population dans le recensement américain.
} Paul Schor

\section{- To cite this version: \\ Paul Schor. Statistiques de la population et politique des catégories aux États-Unis au xixe siècle. Théories raciales et questions de population dans le recensement américain.. Annales de démographie historique, 2003, 105 (1), pp.5-22. 10.3917/adh.105.05 . halshs-01540835}

\section{HAL Id: halshs-01540835 \\ https://shs.hal.science/halshs-01540835}

Submitted on 16 Jun 2017

HAL is a multi-disciplinary open access archive for the deposit and dissemination of scientific research documents, whether they are published or not. The documents may come from teaching and research institutions in France or abroad, or from public or private research centers.
L'archive ouverte pluridisciplinaire HAL, est destinée au dépôt et à la diffusion de documents scientifiques de niveau recherche, publiés ou non, émanant des établissements d'enseignement et de recherche français ou étrangers, des laboratoires publics ou privés. 


\title{
STATISTIQUES DE LA POPULATION ET POLITIQUE DES CATÉGORIES \\ AUX ÉTATS-UNIS AU XIX ${ }^{\mathrm{e}}$ SIÈCLE. THÉORIES RACIALES ET QUESTIONS DE POPULATION DANS LE RECENSEMENT AMÉRICAIN
}

\author{
par Paul SCHOR
}

De la question de l'esclavage à celle de la "nouvelle immigration ", les questions de population aux États-Unis durant la deuxième moitié du XIX ${ }^{e}$ siècle sont indissociables des grands débats qui divisèrent le pays et le recensement fédéral - référence incontestée - y a joué un rôle central. Mais au-delà de l'usage politique des résultats, la construction des catégories statistiques qui ont alimenté ces débats mérite d'être étudiée en détail, tant elle montre à quel point les catégories de l'époque sont tributaires d'une conception de la population en termes anthropologiques et biologiques: infériorité naturelle, hybridité, morbidité et fécondité différentielles, "suicide de la race ", sont en effet les questions auxquelles le recensement a explicitement tenté d'apporter des réponses. On a retenu ici trois cas qui témoignent de cette inscription forte des statistiques démographiques dans des débats tout à la fois scientifiques et politiques: le traitement des statistiques de la folie des noirs libres en 1840, l'impossible métissage entre noirs et blancs en 1850 , et l'invention de l'ethnicité dans les années 1870-1880.
Le recensement fédéral des États-Unis, créé par la Constitution en 1787 et soumis au contrôle du Congrès est, avant d'être un outil de connaissance, un instrument politique. Sa raison d'être était de répartir entre les États fédérés les taxes et les sièges à la Chambre des Représentants, en proportion de leurs populations respectives, ce qui justifie la périodicité du recensement décennal. La Constitution rend obligatoire un recensement de la population tous les dix ans, et automatique la redistribution des sièges dès les chiffres connus. Ce processus, connu en anglais sous le nom d'apportionment, est le plus visible des aspects politiques du recensement, mais il n'est pas le seul ${ }^{1}$. On voudrait montrer que le recensement américain est traversé de considérations politiques qui le constituent; l'allocation des sièges n'est qu'un aspect. On voudrait au contraire s'intéresser à ce que la question de l'apportionment délaisse l'histoire de la production des catégories de la population comme artefacts, produits de la rencontre de considérations politiques, scientifiques, techniques, et pratiques. En effet, la répartition des sièges se fait 
en proportion de la population totale de chaque État, indépendamment de la composition de la population, hommes ou femmes, citoyens ou non-citoyens, blancs ou noirs, adultes ou enfants, résidents légaux ou illégaux. Néanmoins, la Constitution a introduit dès l'origine une distinction essentielle, car prise en compte dans le calcul, la distinction entre les habitants libres et les esclaves, ces derniers ne comptant que comme $3 / 5^{\mathrm{e}}$ des habitants libres. Cette distinction est fondatrice, puisque c'est elle qui est à l'origine du processus de différenciation croissant de la population à travers des catégories toujours plus nombreuses à partir de 1790 .

L'accent mis par les études portant sur le recensement américain, d'une part sur les questions de découpage électoral, d'autre part sur l'allocation de ressources à des catégories de la population mesurées par leurs effectifs dans le recensement, dans le cadre des politiques de discrimination positive conduites depuis les années 1960, a produit un effet d'occultation qui tendrait à faire croire que le recensement en général, et la définition des catégories de la population en particulier, seraient des opérations politiques depuis cette période récente quasi exclusivement. À travers trois exemples d'instrumentalisation politique des catégories au XIX ${ }^{e}$ siècle, dans le contexte des débats sur l'esclavage et sur le métissage au milieu du siècle et sur la " nouvelle immi-gration " au cours des dernières décennies du siècle, on montrera qu'il n'en est rien. D'une part, la constitution des catégories était le fait d'experts sous le contrôle étroit des élus au Congrès, qui jusqu'au recensement de 1930, votaient l'intitulé des questions posées, c'est-à-dire que dans la pratique les titres des colonnes sur les questionnaires corres-pondaient aux termes exacts inscrits dans la loi; d'autre part les résultats en retour, nourrissaient des débats politiques sur l'avenir d'une nation inquiète de la composition future de sa population, des débats qui s'inscrivaient dans le cadre des études sur la population américaine comme composée de races distinctes, inégales, et dont le mélange était craint. Dire que le recensement était une opération politique, ce n'est pas le limiter à ses aspects constitutionnels ou électoraux (Anderson, 1988), mais montrer comment l'ordonnancement même des catégories de la population, justifiées dans le langage de la science de l'époque, pour laquelle la description sociale empruntait à la biologie, ne peut se comprendre qu'à l'intersection de ces domaines que sont la politique, la statistique, la démographie, les sciences sociales, le " racisme scientifique » et les pratiques propres de la communauté des experts. Les exemples qui seront développés montrent l'interaction des décisions politiques, de la pratique des experts et du recours au discours quantitatif pour naturaliser des problèmes sociaux: l'impossibilité pour le recensement d'admettre les erreurs manifestes des statistiques de la folie des noirs libres en 1840, la construction de la catégorie "mulatto " comme moyen non de mesurer mais d'empêcher le métissage entre noirs et blancs, et la recherche de statistiques " ethniques" qui dépassent la simple prise en compte de l'origine nationale.

Instrument politique avant d'être le moyen privilégié de connaître l'évolution de la population américaine, le recensement a toujours été l'objet d'une surveillance attentive de la part du Congrès, et particulièrement de la Chambre des 
Représentants, dont les membres étaient directement concernés par les résultats de l'enquête fédérale. La transformation du recensement en un instrument statistique unique par la richesse et la diversité des informations fournies ne s'est faite que très lentement, car la dimension scientifique du recensement, si elle était défendue dès le départ par certains, venait après sa dimension politique. Les propositions d'enquêtes démographiques avaient d'autant plus de chances d'obtenir la majorité des votes au Congrès ou en commission qu'elles répondaient à une attente des élus.

L'histoire des catégories de la population utilisées par le recensement depuis 1790 est, elle aussi, tributaire de ces contraintes constitutionnelles et politiques. La distinction fondamentale, inscrite dans l'article de la Constitution qui instituait le recensement décennal, était entre les habitants libres et les esclaves, mais on verra que cette distinction de statut était indissociable de la distinction de couleur entre blancs et noirs. Le fait que le mécanisme politique et l'enquête démographique, le statut juridique et la couleur, soient ainsi intimement liés, oblige à considérer le recensement comme une institution hybride, remplissant simultanément plusieurs fonctions différentes, sans que cette distinction soit toujours apparue clairement au législateur ou aux responsables du recensement.

Les statistiques sont en effet un objet singulier d'étude car, par définition, leur ambition est de s'inscrire dans une série et permettre la comparabilité des données à travers le temps. C'est ce qui explique le souhait constant et légitime, des directeurs du recensement d'utiliser les mêmes termes pour désigner les mêmes groupes. Mais, si l'on considère l'enquête statistique qui a lieu tous les dix ans comme un processus d'échange, à travers lequel les habitants donnent aux agents recenseurs des informations quantifiables, tandis que ceux-ci assignent aux individus, au moins durant le temps de l'interaction, des identités non ambiguës, l'objet statistique, replacé dans un contexte précis, est alors dépouillé de la transparence qui était censée le caractériser.

\section{LE RECENSEMENT DE 1840 ET LA SURPRENANTE FOLIE DES NOIRS LIBRES}

Dès le premier recensement, celui de 1790, la loi qui l'organisait, le First Census Act, allait au-delà des exigences de la Constitution, bien que le questionnaire soit très sommaire, ne comportant que 5 catégories. L'article I, section 2, paragraphe 3, de la Constitution prévoyait en ces termes de procéder tous les dix ans à un recensement de la population de tous les États membres de l'Union: «Les représentants et les contributions directes seront répartis entre les États appartenant à cette Union, selon leurs nombres respectifs, qui seront établis en ajoutant au nombre total des habitants libres, y compris ceux qui sont contraints à servir pour une durée déterminée, et, à l'exclusion des Indiens exemptés d'impôts, trois cinquièmes de toutes les autres personnes. »

La Constitution instituait ainsi un recensement dont la fonction était de mesurer ce que l'on a par la suite appelé la population constitutionnelle, base des calculs de répartition, incluant les habitants libres, blancs ou noirs, excluant les Indiens qui, ne payant pas d'impôts, restaient en dehors du corps politique, et ne comptant les esclaves que pour 
trois cinquièmes, ce qui obligeait les agents recenseurs à les dénombrer séparément. Déplaçant la distinction de statut entre esclaves et libres sur un autre plan, connexe mais juridiquement et analytiquement disjoint, la loi de 1790 a introduit dans le recensement une distinction de couleur, qui subsiste aujourd'hui sous la forme de la race, à laquelle tout habitant des États-Unis se rattache naturellement. La distinction entre blancs libres et noirs libres, qui préexiste à la distinction de sexe notamment, s'avère dans les recensements suivants plus importante à certains égards que la distinction entre libres et esclaves. Ainsi les classes d'âge, qui correspondent pour les blancs d'abord à l'âge de servir dans la milice (21 ans) puis au potentiel de participation à la vie active, sont-elles différentes pour les noirs libres, pourtant citoyens, tandis que celles des noirs libres sont, jusqu'en 1830 , les mêmes que celles des esclaves. La comparaison des classes d'âge comparables accentue ainsi l'impression qu'il existe deux populations connaissant des rythmes de vie différents, les blancs d'un côté, les noirs, qu'ils soient esclaves ou non, de l'autre (U.S. Bureau of the Census, $1989)^{2}$.

Le recensement de 1830 , tout en restant dans le cadre limité du recensement par foyer et non par individu, a vu les questionnaires augmentés de rubriques considérées comme des « statistiques sociales " mais enregistrant des faits d'ordre biologique: aveugles, sourds, ou muets, divisés en blancs d'un côté, esclaves et noirs libres confondus de l'autre. À ces questions, le législateur ajouta au recensement de 1840 une question plus incertaine, le nombre de "fous ou idiots " (insane and idiots) qui fut la source d'une des erreurs les plus flagrantes commises lors de la compilation et de la publication des données du recensement américain. En effet, en raison de la disposition des colonnes, un certain nombre de blancs fous furent reportés comme noirs fous, ces colonnes se trouvant l'une à côté de l'autre à la fin du questionnaire, sur un folio de grande taille, comportant 74 colonnes (Cohen, 1982, 187) $)^{3}$. Le résultat de ces erreurs de copies fut que les personnes considérées comme des fous, c'est-à-dire des personnes dépendantes à leur domicile (" at private charge ») ou dans des asiles publics (" at public charge »), furent reportées de telle sorte qu'un grand nombre de fous blancs furent comptés comme noirs dans certains comtés des États du Nord, où tous les noirs étaient libres.

La conclusion logique qui fut tirée de ces chiffres par les responsables du recensement et par les statisticiens du Sud, fut que la liberté rendait les noirs fous, qu'elle leur était naturellement nocive, au point que la proportion de fous parmi les noirs libres augmentait avec la latitude. Dès l'été 1842, un médecin et statisticien du Massasuchetts, spécialiste de statistiques médicales et membre influent de la jeune American Statistical Association, calcula les taux de folie des noirs libres à partir des chiffres $\mathrm{du}$ recensement de la population et obtint ces résultats surprenants: le Maine avait le taux le plus fort, avec un noir sur 14 compté comme fou, tandis qu'en Louisiane seul 1 sur 5650 l'était. Dans l'ensemble, le taux dans le Nord était d'un noir fou ou idiot pour 162,4, tandis que dans le Sud ce taux était d'un pour 1558 , le taux pour les blancs ne variant guère entre le Nord (1 pour 970) et le Sud (1 pour 945,3) (Jarvis, 1842a, 116-121). Cette première constatation était surprenante, mais elle allait dans le 
sens de l'opinion couramment répandue à l'époque selon laquelle les noirs souffraient dans le Nord d'être livrés à euxmêmes, tandis que l'esclavage dans le Sud les préservait de ces soucis.

Mais, dans un deuxième article, en septembre 1842, dans la même revue, Jarvis, revenu plus en détail sur ces chiffres, montrait qu'il s'agissait en fait d'erreurs grossières, puisque l'on trouvait par exemple à Worcester dans le Massachusetts 133 noirs fous, alors que cette ville ne comptait aucun noir, mais que s'y trouvait en revanche un hôpital psychiatrique que Jarvis, lui-même habitant du Massachusetts, et médecin, connaissait bien. Cette constatation l'amena à vouloir vérifier systématiquement d'autres erreurs moins manifestes, et elles étaient nombreuses. Sa conclusion fut sans appel: les erreurs s'étaient produites à Washington, au bureau du recensement, lorsque les relevés des marshals avaient été regroupés et additionnés (Jarvis, 1842b, 281-282).

De fait, les erreurs étaient faciles à trouver pour qui voulait s'en donner la peine. Ainsi, dans l'édition Thomas Allen, qui présentait les données par comtés et par villes principales, on peut trouver des cas d'erreurs flagrantes dans la présentation des données sur la folie des noirs des États du Nord. Dans l'État du Maine, le comté de Somerset compte cinq " colored insane at private charge", et cinq "colored insane at public charge ", ce qui fait dix noirs atteints de folie alors que ce comté ne compte que 16 hommes et 6 femmes noirs. Mais ce sont les données par ville qui montrent le plus grand nombre d'incohérences. Dans la ville de Biddeford, on ne trouve qu'une femme noire, âgée entre 36 et 55 ans, mais deux "insane colored persons". Dans la ville de Danvers, où il n'y a aucun noir, il y a deux noirs fous ${ }^{4}$.
Ces résultats ont donc donné lieu à deux interprétations diamétralement opposées: d'un côté les porte-paroles du Sud, partisans de l'esclavage, trouvant dans le recensement de 1840 la preuve mathématique de l'inadaptation des noirs à la liberté, de l'autre des lecteurs qui contestaient les résultats du recensement, soit par simple rigueur, soit par opposition à l'esclavage. La longue polémique qui a suivi est particulièrement intéressante parce qu'elle constitue la première occasion importante où les données du recensement ont été au cœur d'une controverse politique. Le crédit accordé aux chiffres était plus souvent fonction des choix partisans que des compétences mathématiques des uns et des autres, d'autant plus que le débat tournait autour des chiffres repris par les almanachs et les revues et que les lecteurs et les chroniqueurs prenaient rarement la peine de consulter les volumes complets du recensement. Faire cet effort supposait une suspicion à l'égard des chiffres officiels et des tableaux reproduits dans les revues qui étaient encore rares, et cet effort était d'autant plus grand que ces chiffres, établissant un lien de proportionnalité entre le taux de folie des noirs et leur situation géographique à mesure qu'on allait vers le nord, ne faisaient que conforter des opinions largement répandues.

À partir de l'année 1842, les erreurs avaient été établies pour qui voulait l'admettre, mais les démonstrations rigoureuses de Jarvis et d'autres experts se sont avérées de peu de poids face aux raisons qui poussaient les partisans de l'esclavage à s'arc-bouter sur ces chiffres dans le contexte de défense de "l'institution particulière ".

Les responsables du recensement ont farouchement refusé d'admettre que des 
erreurs avaient pu avoir lieu dans la compilation des données, en premier lieu Weaver, surintendant du recensement en 1840 , parce que sa réputation et ses revenus étaient en jeu. Le nouveau Secrétaire d'État John C. Calhoun, qui avait autorité sur le recensement, était sans doute l'homme politique du Sud le plus important à l'époque, et il ne s'est pas privé d'utiliser largement les chiffres du recensement de 1840 pour défendre l'esclavage (Cohen, 1982, 197).

À partir de ce moment, la question est devenue véritablement une polémique entre élus du Sud et abolitionnistes du Nord au Congrès, tous se réclamant de la vérité scientifique, que peu étaient capables de vérifier par eux-mêmes. L'importance de cette polémique, sur un point finalement secondaire des données du recensement, tient au fait que les données quantitatives étaient désormais devenues un enjeu majeur du débat sur l'esclavage, un débat jusque-là exprimé surtout en termes moraux par les abolitionnistes.

L'autorité des chiffres et celle du recensement officiel des États-Unis ont été invoquées pendant toute la décennie et même au-delà par les défenseurs de l'esclavage, préfigurant l'usage qui sera fait des statistiques de mortalité différentielle des blancs, des noirs et des métis durant les décennies suivantes. La querelle sur les chiffres a permis à une nouvelle rhétorique, mélange d'expertise statistique et d'engagements idéologiques et moraux, de trouver sa place dans le débat politique. Il faut noter que les partisans de l'esclavage adoptaient le plus souvent les positions les plus catégoriques, assénant, chiffres à l'appui, les preuves de la dégénérescence des noirs, tandis que les critiques des résultats du recensement de 1840 gardaient un ton plus mesuré, invoquant une légitimité scientifique dépassionnée.

Les erreurs qui entachaient le recensement de 1840, exposées par Jarvis et d'autres permirent la grande réforme $\mathrm{du}$ recensement en 1849 qui fit du recensement de 1850 le premier recensement à enregistrer les caractéristiques de chaque individu et non plus des familles. Alors que jusqu'à cette date les agents recenseurs portaient dans les différentes colonnes le nombre de personnes de sexe masculin ou féminin, de telle classe d'âge, de libres ou d'esclaves, désormais chaque individu figurait sur une ligne distincte avec ses propres caractéristiques, parmi lesquelles la couleur occupait une place essentielle.

\section{COULEUR, RACE ET ORIGINE DES ESCLAVES ET DES LIBRES : LES STATISTIQUES COMME REFUS DU MÉTISSAGE}

Deux types de questions devant figurer sur les questionnaires de 1850 ont donné lieu à des débats très vifs au Congrès en 1850, aussi bien au Sénat qu'à la Chambre des Représentants: celles portant sur l'esclavage et celles portant sur la couleur des habitants des États-Unis, distingués en trois catégories, blancs, noirs et mulâtres (mulatto). Les élus du Sud étaient partagés entre le désir de connaître statistiquement la population servile, notamment dans l'espoir de démontrer sa longévité supérieure à celle des noirs libres du Nord, et la suspicion à l'égard d'une "inquisition " du gouvernement fédéral sur cette question qui divisait l'Union et donc le Congrès. Après avoir obtenu, que sur le questionnaire simpli- 
fié qui recensait les esclaves, ils ne soient pas identifiés par leurs noms, comme les habitants libres, mais par des numéros, et que leur lieu de naissance n'apparaisse pas non plus, les élus du Sud ont porté leurs objections sur la catégorie de mulatto, trace quantifiée du mélange entre noirs et blancs (Schor, 2001, 7883). Le sénateur Underwood, qui défendait le projet de loi préparé par un comité composé d'experts, a justifié l'inclusion de cette catégorie, d'abord sur le questionnaire $\mathrm{n}^{\circ} 2$, celui des esclaves, comme permettant de mesurer "le degré d'éloignement par rapport à la race pure ${ }^{5}$ (U.S. Congress, 1850, XXI, 674). Il a notamment indiqué l'intérêt de pouvoir établir des tables comparatives entre les deux races, noire et blanche, étant entendu que les mulattoes ne constituaient pas une race intermédiaire mais un sous-ensemble de la population noire, ce que leur prise en compte dans les questionnaires comme dans les volumes publiés du recensement montre sans équivoque. Mais il était également conscient de la charge polémique du principe même de ce genre d'enquête: "Maintenant la question est: êtes-vous prêts à recueillir toutes ces informations, dans le but d'établir les lois de longévité entre les deux races, les proportions de sang et les autres lois physiques des races?"

Et répondant à de nouvelles objections, notamment à celle, classique, qui rappelait que le but du recensement n'était pas de se livrer à un travail scientifique mais de compter simplement les habitants, Underwood a défendu l'idée qu'il ne s'agissait là que de rassembler des faits simples, relevant du sens commun, rejetant implicitement l'idée que le recensement contribuerait en nommant et en comptant à renforcer ces distinctions qui semblaient naturelles: «Ce n'est pas du tout une question d'investigation scientifique, mais une simple enquête sur des faits - à savoir si un individu est un quarteron [quadroon], un mulâtre [mulatto] ou a une proportion quelconque de sang. [...] Et certainement, le public a l'intérêt, de même que le gouvernement, de connaitre la condition relative réelle des différentes classes de la population. " (U.S. Congress, 1850, XXI, 675)

Mis en difficulté par les élus du Sud qui menaçaient de revenir à loi de 1840 et donc de supprimer toutes les innovations du recensement de 1850, Underwood a dû en appeler à l'autorité d'un médecin sudiste respecté par les partisans de l'esclavage et de l'infériorité naturelle des noirs, pour pouvoir convaincre les sénateurs que les résultats n'affaibliraient pas nécessairement la cause des esclavagistes: "Il croyait qu'une certaine classe de personnes de couleur avait moins d'enfants qu'une certaine autre classe; et il croyait que la durée moyenne de vie des enfants de la classe la plus sombre était plus longue que celle des enfants de la classe de couleur plus claire, ou des métis [mixed]. "

Du coup, une partie des sénateurs du Sud sont revenus sur leur opposition, commentant les différences entre les métis et les noirs. Ainsi, le sénateur Dayton, reprenant ce que le sénateur King avait dit sur le fait que les "vrais noirs " étaient plus résistants, a conclu " en un mot, le mulâtre est à un certain degré un hybride! ", par quoi on doit comprendre qu'il s'agit d'une espèce moins apte à la reproduction (" moins prolifique ») et moins forte. Rassurés sur l'utilisation potentielle des données, qui devaient pour eux démonter l'infériorité biologique des produits du métissage, 
les élus sudistes ont finalement accepté ces innovations. Ces discussions de 1850 se déroulaient dans un contexte scientifique nouveau: alors que, durant la première moitié du siècle, l'interprétation rigoureuse de la doctrine chrétienne empêchait de douter de la création d'un homme unique par le créateur, ce verrou théologique et idéologique avait sauté. Il était désormais possible de professer le polygénisme sans être immédiatement accusé de crime contre la religion (Haller, 1971; Stanton, 1960). La question du métissage reviendra après l'abolition de l'esclavage puisque cette catégorie de mulatto divise la population noire dans tous les recensements de 1850 à 1920, à l'exception de celui de 1900, la proportion de mulattoes passant progressivement de $11,2 \%$ des noirs en 1850 à $20,9 \%$ en 1910 et $15,9 \%$ en $1920^{7}$. Cette dernière variation a fourni au bureau du recensement la raison de renoncer à cette catégorie après 1920 , au motif que les agents noirs engagés en 1910 avaient vu davantage de mulattoes que les agents recenseurs blancs chargés des mêmes comtés en 1920. En 1920, cette catégorie n'était plus nécessaire, du fait de l'intériorisation de la one drop rule par les blancs comme par les noirs, cette règle, qui faisait qu'une goutte de sang noir faisait un noir, ne justifiait plus le maintien d'une distinction dont la principale fonction avait été de conserver au sein de la population noire les personnes qui auraient pu dans un autre contexte être considérées comme appartenant à un groupe intermédiaire, voire dénombrées comme blanches, mettant en danger la pureté de la race blanche, que le recensement avait aussi pour tâche de préserver.

Il faut remarquer que la catégorie de mulatto a été utilisée en 1850 et 1860 sans qu’aucune définition ni instruction ne soit fournie par le Census Office, ce qui laissait les agents recenseurs, dans le cas des libres, et les propriétaires, dans le cas des esclaves, déterminer qui était noir et qui était mulatto ${ }^{8}$.

Après le recensement de 1860, alors que l'émancipation de près de quatre millions d'esclaves semblait en vue, le directeur du recensement de $1860 \mathrm{a} \mathrm{pu}$ trouver dans les chiffres de quoi apaiser les craintes suscitées par cette perspective: l'extinction naturelle de la race noire, par absorption ou par abâtardissement. Dans son introduction au volume du recensement de la population en 1860 , J. C. G. Kennedy a ainsi pu écrire en 1864, avant même la fin de la guerre et l'abolition: «Si nous regardons l'avenir, il semble, dès lors, tout à fait rationnel de conclure que nous n'avons pas besoin d'attendre des siècles pour que se développe le fait que la race blanche n'est pas plus favorable à la présence de la race africaine en son sein qu'elle ne l'a été à la perpétuation de la présence de la race indienne à ses frontières, et que, comme cela a été le cas dans tous les autres pays de ce continent où les noirs ont jadis été nombreux, la population de couleur en Amérique, qu'elle soit esclave ou libre, partout où elle est nettement subordonnée en nombre et en condition à la race blanche, est vouée à une absorption relativement rapide ou à l'extinction. Comment ce résultat pourrait être évité, au moins partiellement, ce sera à d'autres d'en juger, estimant pour notre part notre devoir accompli en développant les faits, tels qu'ils ressortent des chiffres du recensement sur la période écoulée. " (U.S. Census Office, 1864, p. XII)

Ce thème de l'extinction naturelle des noirs libres, reprenant sous une autre 
forme le raisonnement explicatif de la folie des noirs libres en 1840, tirera une légitimité scientifique de sa présence dans les publications officielles du recensement américain, et il faudra plusieurs décennies pour que tous se rendent à l'évidence de la permanence de la population des descendants d'esclaves, dont la part relative a diminué principalement en raison de l'apport considérable de l'immigration européenne dans la deuxième moitié du $\mathrm{XIX}^{\mathrm{e}}$ siècle, tandis que la population noire n'augmentait que par accroissement naturel ${ }^{9}$. Progressivement, à mesure que la part relative de la population noire se stabilisait et déclinait lentement, les interprétions biologiques de la composition de la population américaine se sont reportées sur une autre crainte d'hybridation néfaste, le mélange de races européennes inférieures avec les races anglo-saxonnes, pendant de l'absorption des noirs, tandis que le thème de l'extinction de la race noire trouvait un écho inattendu dans la théorie du suicide de la race, dont le directeur des recensements de 1870 et 1880, Francis A. Walker fut un des champions.

\section{L'INVENTION DE L'ETHNICITÉ DANS LES RECENSEMENTS DE 1870 ET 1880}

La prise en compte croissante de l'origine nationale par le recensement américain à partir de 1850 permet de situer dans la deuxième moitité du siècle, et plus précisément dans les recensements de 1870 et 1880 l'invention de l'ethnicité, c'est-à-dire un intérêt porté aux habitants des États-Unis pour lesquels l'origine nationale, de première ou de deuxième génération, est une variable explicative de leur position dans la société d'accueil (Conzen et al., 1992). Deux principes peuvent être dégagés de la façon dont le recensement a pris en compte les origines étrangères: d'une part, ce sont les immigrés qui intéressent le recensement, et non les étrangers, d'autre part, et c'est pour cela que l'on parle de l'invention de l'ethnicité et non de statistiques d'immigration, l'objet qui est construit tente de s'affranchir des nationalités d'origine telles que définies par le droit pour saisir la stratification sociale en fonction de critères conçus comme culturels et biologiques.

Les recensements antérieurs n'ont pris en compte qu'imparfaitement la diversité des origines des Américains: le recensement de 1820 a distingué les étrangers non naturalisés, foreigners not naturalized, du reste de la population; celui de 1830 a poursuivi en utilisant le terme alien, c'est-à-dire les étrangers au sens juridique du terme, citoyens d'un autre pays, mais aucune question ni sur la nationalité ni sur le lieu de naissance ne figurait dans le recensement de 1840 qui avait pourtant vu une multiplication de ses rubriques. À l'inverse, le recensement de 1850 , le premier à recueillir des données par individu a introduit la question du lieu de naissance, qui permettait de connaître les migrations internes et internationales. Comme on l'a vu, les sénateurs sudistes avaient obtenu la suppression de cette question pour les esclaves, ce qui empêche de reconstituer les parcours des esclaves, qu'il s'agisse de la traite officiellement interdite après 1808 , ou de l'éclatement des familles.

Avec le recensement de 1850, qui révèle la présence de 2244602 immigrés, soit $9,7 \%$ de la population totale, les États-Unis prennent conscience de l'im- 
portance quantitative de l'immigration, qui est pour beaucoup une surprise. Le recensement de 1860 marque le début d'une longue période durant laquelle la part des immigrés oscille entre 13,2 et $14,7 \%$, pour décliner avec les lois restrictives des années 1920, et ne retrouver de tels niveaux qu'à la fin du $\mathrm{XX}^{\mathrm{e}}$ siècle. Les rapports du directeur du recensement mettent aussi en valeur la part des différents pays, ainsi l'Irlande représente à elle seule le pays de naissance de 961719 habitants des États-Unis en 1850, soit $43,5 \%$ des immigrés (U.S. Congress, 1853, 10-14; Schor, 2001, 220-226).

Les recensements de 1850 et 1860 se bornaient à prendre en compte le lieu de naissance, sans considération de nationalité, alors que les recensements de 1820 et 1830 avaient uniquement recueilli le nombre d'étrangers. Il faudra attendre 1890 pour que soit posée une question sur la naturalisation des habitants nés à l'étranger. Car ce qui préoccupe les responsables du recensement, et au-delà, les responsables politiques, ce n'est pas la citoyenneté ou la nationalité au sens juridique du terme, mais les origines des immigrés. Dès les résultats du recensement de 1860 connus, le directeur du recensement, Kennedy, déplore que le pays de naissance masque dans un certain nombre de cas la race des immigrés européens. Les spécialistes de la population emploient à l'époque plus facilement le terme de race pour parler des races européennes, tandis que le terme de couleur renvoie à la distinction entre blancs et noirs. S'interrogeant sur la "fusion des races ", Kennedy insiste sur les différences culturelles que le recensement ne prend pas directement en compte, ainsi des Alsaciens et Lorrains, qui représentent une bonne part des immigrés originaires de France en 1860, mais « qui sont en réalité Allemands par leur origine, et parlent la langue allemande, bien qu'ils aient été comptés indistinctement avec les autres natifs de France. »(U.S. Census Office, 1684, 1, XXXII). Sur un autre plan, dans la prise en compte des réponses, leur compilation, et leur publication, le Census Office va démontrer qu'il s'intéresse à des origines nationales qui ne renvoient pas à des nations existantes. Conformément à la théorie des races alors dominante, le recensement avait distingué les Anglais des Écossais, Gallois et Irlandais. Mais dès la publication des résultats de 1860 , il introduit une autre innovation: les habitants des États-Unis nés " dans l'ancien royaume de Pologne » seront comptés comme de naissance polonaise, alors qu'à cette date ils n'ont plus de pays ni de gouvernement. Le recensement reconnaissait ici manifestement la présence d'une communauté d'origine polonaise sur le sol américain, ce qui fondait l'existence de cette catégorie, maintenue de 1860 à 1890 . Il semble que les nombres aient eu leur importance, puisque, au recensement de 1870, "les quelques cas où le lieu de naissance a été reporté comme "Finlande" ont été classés comme "Russie". " (U.S. Bureau of the Census, 1922, II, 688; U.S. Census Office, 1872, I, 317). Le cas de la Pologne a été également l'occasion d'une prise en compte partielle de la langue maternelle, qui ne sera généralisée à tous les immigrés qu'en 1910. En 1900 les instructions aux agents recenseurs indiquaient pour le lieu de naissance: «Dans le cas où la personne parle polonais, comme la Pologne n'est pas actuellement un pays, demandez si le lieu de naissance se trouvait dans ce qui est maintenant connu sous le nom de Pologne allemande ou Pologne autrichienne ou Pologne 
russe, et reportez la réponse appropriée comme Poland (Ger.), Poland (Aust.) ou Poland (Russ.)." (U.S. Bureau of the Census, 1989, 42)

Pour être statistiquement " né en Pologne » dans le recensement américain de 1900, le fait de la naissance dans ce territoire précis ne suffisait pas, il fallait aussi être de langue polonaise, c'est-àdire appartenir au groupe majoritaire, dont la culture identifiait aux États-Unis les immigrés "polonais ». Il y a donc là une première transformation des statistiques de lieu de naissance en statistiques ethniques, c'est-à-dire en statistiques qui n'ont pas pour but de cerner une population dans le pays d'origine, mais de distinguer une population dans le pays d'accueil. Le cas des Polonais, sans doute mieux organisés, est particulier puisqu'ils ont obtenu du recensement fédéral une forme de reconnaissance qui leur était déniée par le droit international, dès 1864. À leur suite, on trouvera à partir de 1870 comme lieu de naissance la Bohême et deux possibilités pour le Canada à partir de 1890, Canada-English et Canada-French, une division qui n'est pas strictement géographique mais tient compte de "l'extraction » de la personne, et est donc aussi une construction de l'origine ethnique ${ }^{10}$.

Cette volonté de connaître les caractéristiques de la population d'origine étrangère explique une transformation plus importante, l'invention de la deuxième génération, ou foreign stock, les Américains dont l'un ou les deux parents étaient immigrés, au recensement de 1870 .

Dans un contexte d'inquiétude croissante à l'égard de l'immigration, le rapporteur du projet de loi de recensement pour 1870 en a exposé très claire- ment les motifs: "Il a été instamment recommandé, et à juste titre, que, à la question sur le lieu de naissance, soit ajouté le lieu de naissance du père et de la mère de chaque personne. Ceci nous permettrait d'établir la fécondité relative de nos populations américaine et née à l'étranger. On a récemment soutenu que l'ancien taux de croissance parmi nos populations natives diminue rapidement. Si cela était vrai, ce fait d'une importance vitale doit être établi et son amplitude complète et sa signification déterminées. » (U.S. Congress, 1870, $\left.181^{11}\right)$

En fait, en 1870, le lieu de naissance des parents n'était pas reporté, seule la naissance étrangère ou non des parents était indiquée, sans préciser le pays, ce que n'a pas manqué de déplorer le rapport du recensement de 1870 (U.S. Census Office, 1872, 1, XXIX). Le recensement de 1880, dont le directeur, Francis Amasa Walker, était déjà celui de 1870, ajoutera le lieu de naissance de chaque parent, ce qui permettait de croiser les origines étrangères distinctes des parents, une opération développée à partir de 1880 sous la forme de tableaux croisés présentant, par pays de naissance du père, les pays de naissance les plus nombreux pour les mères correspondantes. Dans les brefs commentaires, le rédacteur n'a pas manqué d'évoquer «le nombre étonnamment faible d'hommes irlandais mariés avec des femmes allemandes. " (U.S. Census Office, 1883, 1, $\left.677^{12}\right)$. Ces tabulations croisées, même si elles ne concernent pas toutes les personnes de parents nés à l'étranger, permettent d'avoir une idée de l'endogamie de la deuxième génération d'origine immigrée.

En revanche Walker a retiré du recensement de 1880 la proposition de question 
sur la naturalisation des personnes nées à l'étranger, estimant que la réponse était trop incertaine, d'autant plus que les agents recenseurs avaient souvent comme interlocuteurs les femmes, ce qui était pour lui un obstacle pratique, en raison de leur manque d'intelligence, répondant ainsi au Secrétaire à l'Intérieur : "Combien de femmes américaines peuvent dire quelle est la procédure légale par laquelle un étranger devient un citoyen? Et il faut garder à l'esprit que l'agent recenseur, même s'il comprend la question lui-même, n'a pas de moyen pratique disponible pour s'assurer luimême du savoir de la femme ${ }^{13}$."

Cet intérêt pour les parents de naissance étrangère traverse les enquêtes et les publications du recensement à partir des années 1860, la place de ces questions sur les questionnaires et dans les volumes publiés ne cessant de croître. Comme l'avait remarqué le rapporteur du projet de 1869 , cité plus haut, l'inquiétude se cristallisait sur les taux de fécondité différentiels de la population native, c'est-à-dire de parents nés aux États-Unis et de la population immigrée de la première ou de la deuxième génération, le foreign stock créé en 1870. Cette théorie, connue par la suite sous le nom de "suicide de la race ", reposait sur l'idée, inspirée de darwinisme social, que dans la compétition économique avec les immigrés acceptant des salaires inférieurs, les Américains de vieille souche répondaient en réduisant leurs naissances, ou pour reprendre l'expression de Walker qui après son départ du recensement publiera de nombreux articles sur ce thème, le " principe de population " était atteint: "Dès la première apparition en grand nombre des étrangers aux États-Unis, leur taux de croissance a été plus grand que parmi ceux qu'ils ont trouvés ici; et cette disproportion a continué, depuis, à s'accroître. Mais est-ce que ce résultat est dû à un déclin de la vitalité physique et de la vigueur reproductrice de cette partie de la population que nous appelons, par comparaison, américaine, ou est-ce dû à d'autres causes, peut-être à l'apparition des étrangers eux-mêmes? [...] L'arrivée des étrangers, à l'époque et selon les circonstances, a été un choc pour le principe de population parmi l'élément natif. Ce principe est toujours très sensible, similaire aux conditions sentimentales et économiques. Et il faut noter, en passant, que non seulement le déclin de l'élément natif, dans l'ensemble, s'est produit de façon à correspondre singulièrement à l'excès d'arrivées étrangères, mais il s'est produit principalement dans ces régions où les nouveaux arrivants se dirigeaient le plus librement. " (Walker, 1891, 634-643)

La force de l'argumentation de Walker était de combiner analyse statistique, psychologie des masses et d'aller dans le sens d'un sentiment alors largement répandu de danger de disparition de la civilisation américaine submergée par la "nouvelle immigration». Ces arguments ont été depuis réfutés, y compris sur la base des données sur la fécondité, qui attestaient bien de différences entre natives et foreigners, mais ces différences restaient faibles ${ }^{14}$.

\section{Conclusion}

Ces trois exemples montrent comment le travail scientifique du recensement était indissociable des enjeux politiques des questions de population. Les statistiques sur la folie des noirs et le refus de corriger les erreurs, la construction d'une souspopulation noire métissée pour observer sa 
dégénérescence et nier la possibilité d'un groupe intermédiaire, et la constitution du foreign stock, montrent l'inscription du recensement dans l'élaboration d'une sociologie des populations qui emprunte beaucoup aux modèles biologiques du racisme scientifique aussi bien qu'à ses représentations populaires. Alors que l'introduction des catégories doit souvent beaucoup au contexte politique immédiat, qu'expriment les amendements des membres du Congrès, le recensement, outil statistique dont l'inertie est constitutive, à des fins de comparabilité, conserve et transforme ces catégories qui grâce à lui survivent durant des décennies au contexte de leur introduction. Ainsi, l'abolition de l'esclavage n'entraîna nullement la disparition des catégories de couleur qui avaient à cette date acquis une légitimité scientifique incontestée. Il en va de même de l'évolution de la prise en compte du lieu de naissance des personnes, puis de leurs parents, en statistiques de l'ethnicité, qui visaient à faire apparaittre les lignes d'organisation sociale et spatiale des descen- dants des immigrés au sein de la société américaine; introduites pour nourrir les thèses des partisans de la restriction de l'immigration, elles ont permis à des minorités, slaves en particulier, d'attendre du recensement qu'il les distingue, par la langue notamment, des empires européens qui régnaient sur leurs régions d'origine. Le recensement se trouvait ainsi à l'intersection de nombreuses demandes contradictoires, qu'il s'agisse des catégories ou du traitement des résultats, et de ce point de vue, l'histoire de la construction de ces données semble indispensable à la compréhension des chiffres officiels de la population américaine.

\section{Paul SCHOR \\ U.F.R. d'Études Anglo-Américaines, Université de Paris- $X$, 200, avenue de la République, Nanterre, 92000. email:paul.schor@u-paris10.fr}

\section{Notes}

1. Les aspects mathématiques des différentes méthodes utilisées ont été étudiés par Balinski et Young (1982). La question de l'allocation des sièges est depuis les années 1930 liée à celle de l'utilisation ou non d'échantillons pour corriger les chiffres de la population physiquement rencontrée, le sampling, jusqu'ici rejetée par le pouvoir politique. La Constitution emploie l'expression " actual enumeration " ce qui fonde l'argument constitutionnel des adversaires de l'ajustement. Les élus républicains estiment souvent que la correction réintroduirait une population d'habitants pauvres et appartenant aux minorités, dont ils supposent qu'ils confieraient leurs votes de préférence aux démocrates. En réalité, les sièges étant alloués aux États et non aux districts sousénumérés, cette conséquence est loin d'être certaine. Pour la période récente et notamment les problèmes posés par la préparation du recensement de 2000, voir (Skerry, 2000).

2. Les questionnaires sont reproduits en fac simile dans U.S. Bureau of the Census (1989). Les cinq catégories du recensement de 1790 étaient après les noms des chefs de famille: hommes blancs libres de 16 ans et plus, dont " chefs ", hommes blancs libres de moins de 16 ans, femmes blanches libres, dont "chefs ", tous les autres libres, esclaves. Pour un commentaire plus détaillé de ces évolutions, voir (Schor, 2001, 23-47).

3. Le récit le plus détaillé sur le recensement de 1840 et ses erreurs est donné par P. Cohen (Cohen, 1982, 175-204). Les erreurs ont été aggravées par un conflit entre deux imprimeurs, 
celui de Weaver, responsable du recensement en 1840, renvoyé par le Congrès, et celui choisi par la nouvelle majorité au Congrès pour finir le travail, ce qui a produit deux éditions concurrentes du recensement de 1840, dans lesquelles les erreurs sont différentes. La lecture des rapports du Congrès sur le conflit entre les imprimeurs a permis à Patricia Cohen de comprendre à quel stade les erreurs s'étaient glissés dans les résultats publiés, puisqu'ils fournissent une source exceptionnellement détaillée sur les étapes du travail de compilation. Elle a montré que les erreurs provenaient largement de la transcription des questionnaires originaux sur des listes préparées pour les volumes publiés dont les en-têtes de colonnes étaient légèrement différents, alors que l'état des connaissances mathématiques de l'époque ne permettait pas de comprendre comment des erreurs sur un nombre limité d'individus avaient été amplifiées; les critiques de l'époque avançaient l'hypothèse de la fraude ou d'erreurs au moment de l'enregistrement.

4. Ces exemples sont tirés des pages 5, 7, 9 et 11 de l'édition Allen du Compendium of the Enumeration of the Inhabitants and Statistics of the United States, as obtained at the Department of State, From the returns of the Sixth Census, by Counties and principal Towns, exhibiting the Population, Wealth, and Resources of the Country. Prepared at the Department of State. Washington, Printed by Thomas Allen. 1841. N'ont été retenues ici, à titre d'exemple, que les premières erreurs grossières que j'ai trouvées en consultant ce volume; on aurait pu en inclure bien d'autres pour tous les États du Nord, comme celles relevées par Jarvis. Un exemplaire de ce volume très rare se trouve à la Bibliothèque Nationale de France, cote Fol-PB-967.

En vérité, le volume a été publié en 1842 mais Allen l'a antidaté pour augmenter ses chances dans la dispute pour le contrat officiel de publication du recensement fédéral, (Cohen, 1982, 260).

5. Séance du Sénat, 9 avril 1850.

6. Italiques dans le texte. Par «proportion quelconque de sang " Underwood parle de degré de mélange entre sang blanc et sang noir. L'expression " any proportion of blood" est la définition de la one drop rule: une proportion si infime soit- elle de sang noir, fait d'un individu un noir. Les " différentes classes " de la population sont évidemment les différentes races. L'euphémisation était toujours de rigueur dans le discours politique sur l'esclavage, comme on l'a vu avec l'article I de la Constitution qui n'évoque les esclaves que comme "toutes les autres personnes ". Cette réticence à nommer se retrouve dans le recensement de 1850, qui, s'il emploie le mot "slave ", n'enregistre pas les noms des esclaves mais les renvoie à leur statut de propriété en les désignant par des numéros.

7. Contre l'avis des responsables du recensement, le Congrès, sur amendement d'un élu sudiste, a ajouté au recensement de 1890 des distinctions plus fines encore, entre noir, mulâtre (mulatto), quarteron (quadroon) et octavon (octoroon). Le représentant Wheeler de l'Alabama avait introduit son amendement pour " obtenir et démontrer les effets physiques sur les descendants issus du mélange des espèces humaines " (Nobles, 2000, 55-56). Obligé par la loi à publier ces résultats, le Census Office n'a donné ces résultats qu'au niveau national et a présenté dans tous les autres tableaux tous les noirs fondus en une seule catégorie, estimant que ces résultats n'avaient pas de valeur scientifique, réduisant à néant les espoirs de voir confirmées par les chiffres les théories sur le métissage. Au recensement suivant, en 1900 , toutes les distinctions au sein de la population noire ont été supprimées (Schor, 2001, 178-186).

8. Les variations des proportions relatives de noirs (black) et de mulâtres (mulatto) ne peuvent s'expliquer autrement dans le cas des esclaves, alors que les agents recenseurs étaient les mêmes pour différentes propriétés d'une même région. Les données confirment ce que l'on sait par ailleurs, à savoir qu'il n'y avait pas d'interaction entre les agents et les esclaves, mais entre les agents et les propriétaires ou les régisseurs, qui répondaient aux questions portant sur les esclaves. Sur les incertitudes de ces données et ce qu'elles permettent néanmoins de savoir sur l'étendue des unions interraciales, voir les travaux impulsés par Robert Fogel (Fogel et Engerman, 1974, I, 131-133, II, 108-110; Steckel, 1980, 260). Les recensements de 1850 et 1860 étant les seules données documentant la descendance des unions mixtes parmi les esclaves à l'échelle nationale, ces chiffres ont fait l'objet de 
nombreuses interprétations divergentes (Williamson, 1980; Berlin, 1974; Mills, 1981). Sur l'importance de l'interaction et de la perception différente de la couleur par les agents recenseurs, voir (Schor, 2001, 100-124; Washington, 1997, 170-212).

9. «Le point de vue de Kennedy, confirmé par la suite par des analyses fautives dans les neuvième et dixième recensements de 1870 et 1880 , corroboré par les croyances des médecins et les enquêtes de compagnies d'assurance américaines, par les données statistiques de l'U.S. Army, ainsi que par d'innombrables rapports médicaux, a précipité la croyance en l'inévitable extinction des Noirs. " (Haller, 1971, 40)

10. Les instructions aux agents de 1890 indiquaient: "Si la personne est née au Canada ou à Terre-Neuve, inscrivez les mots "English" ou "French" après le lieu de naissance, de manière à distinguer parmi toutes les personnes nées en Amérique britannique celles qui sont d'extraction française ou anglaise respectivement. Ceci est une exigence très importante, et doit être scrupuleusement observée dans chaque cas et la distinction devra être soigneusement faite. " (U.S. Bureau of the Census, 1989, 37)

11. Séance du 16 décembre 1869, House of Representatives.

12. Le texte indique aussi qu'il n'est pas impropre de parler d'Allemand même pour une personne née aux États-Unis, si elle a été élevée dans une famille allemande, mais que « ce n'est pas le point de vue de la loi du recensement "; le recensement parle de «foreigners " (étrangers) pour désigner la naissance étrangère. Ainsi " les statistiques de la deuxième génération d'étrangers (foreigners) seront trouvées plus loin dans une autre série de tableaux (Foreign Parentage) "(Id. p. 460). Le recensement avoue ainsi devoir se plier à la rigueur du vocabulaire juridique, mais que l'objet qu'il construit correspond davantage aux catégories mentales populaires.

13. Réponse écrite de Walker, lue devant la Chambre des Représentants le $1^{\text {er }}$ avril 1880. Congressional Record. House. "The Census". 46 Congress, $2^{\text {nd }}$ session, p. 2033. Cité par D. Magnuson (1995, 59-60).

14. Miriam L. King a repris les chiffres de Walker et refait les calculs à partir des données de l'échantillon IPUMS de 1900 et montré que le " race suicide " n'avait jamais eu lieu mais que les différences de fertilité entre natives et foreigners étaient faibles et étaient compensées par les différences de fertilité entre immigrés de la première et deuxième génération. Elle conclut que la croyance selon laquelle les immigrés et leurs enfants avaient une fertilité plus élevée que les native-born of native parentage reflétait les craintes nativistes davantage que les réalités démographiques (King, 1990).

\section{RÉFÉRENCES BIBLIOGRAPHIQUES}

Anderson, Margo (1988), The American Census. A Social History, Newhaven, CT, Yale University Press.

Balinski, Michel, Young, H. Peyton (1982), Fair Representation: Meeting the Ideal of One Man, One Vote, New Haven, CT, Yale University Press.

Berlin, Ira (1976), Slaves Without Masters, The Free Negro in the Antebellum South, New York, Vintage Books.

Compendium of the Enumeration of the Inhabitants and Statistics of the United States (1841), Washington, DC, Thomas Allen.
Conzen, Kathleen Neils, Gerber, David A., MorawsKa, Eva, PozzetTA, George E., et VECOLI, Rudolph J. (1992), "The Invention of Ethnicity: A Perspective from the U.S.A.", Journal of American Ethnic History, Vol.12, 1, 3-41.

Fogel, Robert et EngERMan, Stanley (1974), Time on the Cross, Vol. 1, The Economics of American Negro Slavery, Vol. 2, Evidence and Method, Boston, Little Brown.

Haller, John S. (1971), Outcasts from Evolution: Scientific Attitudes of Racial Inferiority, 1859-1900, Urbana, University of Illinois Press. 
JARVIS, Edward (1842a), "Statistics of Insanity in the United States", Boston Medical Journal, 27, 116-121.

JARVIS, Edward (1842b), "Statistics of Insanity in the United States", Boston Medical Journal, 27, 281-282.

KING, Miriam L. (1990), The Quantum of Happiness: The Politics of American Population Debates, 1850-1930, Ph. D, University of Pennsylvania.

Magnuson, Diana Lynn (1995), The Making of a Modern Census: the United States Census of Population, 1790-1940, Thèse Doct. University of Minnesota.

MilLs, Gary B. (1981), "Miscegenation and the Free Negro in Antebellum 'Anglo' Alabama: A Reexamination of Southern Race Relations", Journal of American History, Vol. 68, N 1, 16-34.

Nobles, Melissa (2000), Shades of Citizenship. Race and the Census in Modern Politics, Stanford, Stanford University Press

SCHOR, Paul (2001), Compter et classer. Histoire des catégories de la population dans le recensement américain, 1790-1940, Thèse de doct., Paris, EHESS.

Skerry, Peter (2000), Counting on the Census? Race, Group Identity and the Evasion of Politics, Washington, DC, Brookings Institution.

STANTON, William R. (1960), The Leopard's Spots. Scientific Attitudes Towards Race in America, 1815-1859, Chicago, University of Chicago Press.

STECKEL Richard H. (1980), "Miscegenation and the American Slave Schedules", Journal of Interdisciplinary History, XI, 2, 251-263.

U.S. Bureau of the Census (1922), The Fourteenth Census of the United States, Washington, DC, Government Printing Office.

U.S. Bureau of the Census (1989), 200 Years of U.S. Census Taking: Population and Housing questions, 1790-1990, Washington, DC, Government Printing Office.
U.S. Census OfFice (1864), Population of the United States in 1860; Compiled from the Original Returns of the Eighth Census, under the direction of the Secretary of the Interior, by Joseph C. G. Kennedy, Superintendent of Census, Washington, DC, Government Printing Office.

U.S. Census Office (1872), Ninth Census: The Statistics of the Population of the United States, embracing, The Tables of Race, Nationality, Sex, Selected Ages, and Occupations. To which are added The Statistics of School Attendance and Illiteracy, of Schools, Libraries, Newspapers and Periodicals, Churches, Pauperism and Crime, and of Areas, Families, and Dwellings. Compiled from the original returns of the Ninth Census (June 1, 1870) under The Direction of the Secretary of the Interior, Francis A. Walker, Superintendent of Census, Washington, DC, Government Printing Office.

U.S. Census Office (1883), Statistics of the Population of the United States at the Tenth Census (June 1, 1880), Washington, DC, Government Printing Office.

U.S. Congress (1850), The Congressional Globe, Washington, DC, Blair \& Rives.

U.S. Congress (1870), The Congressional Globe, $41^{\text {st }}$ Congress, $2^{\text {nd }}$ Session, Washington, DC.

U.S. CONGRESS (1853), The Seventh Census. Report of the Superintendent of the Census For December 1, 1852; To which is appended the Report for December 1, 1851, Washington, DC, Robert Armstrong, Printer.

WASHINGTON, Mary Lynn (1997), White, Black, or Mulatto: A Sociological Exploration of the Meaning of Racial Classification in the United States Census of 1880 , Ph.D., Johns Hopkins University.

WALKER, Francis A. (1891), "Immigration and Degradation", The Forum, 11, 634643.

Williamson, Joel (1980), New People. Miscegenation and Mulattoes in the United States, New York, The Free Press. 


\section{RÉSUMÉ}

Le recensement américain de la population qui a lieu tous les dix ans depuis 1790, a été créé par la Constitution pour répartir les sièges de Représentants en proportion de la population des États. Pour cette raison le Congrès a toujours exercé un contrôle sur les questions posées, et la dimension politique du recensement était forte. À partir de trois cas, on montre comment le recensement était à l'intersection de différents niveaux politiques, scientifiques, techniques; en aval les résultats nourrissaient les débats, en amont les catégories étaient aussi conçues en fonction du contexte politique. Le traitement des statistiques de la folie des noirs libres en 1840, la création d'une catégorie de métis (mulatto) en 1850 pour maintenir les métis au sein de la population définie comme noire et dans l'espoir de documenter la dégénérescence des "hybrides", enfin la volonté à partir de 1870 de définir les descendants des immigrés par des traits hérités et non par le seul lieu de naissance, montrent comment les questions politiques de population étaient pensées en termes biologiques également.

\section{SUMMARY}

The American census, taken every ten years since 1790 , was created by the Constitution to apportion the seats of Representatives in proportion of the population of the States. Therefore, Congress maintained a firm control on the questions asked, and the political dimension of the census was important. Looking at three cases, I show how the census was located at the intersection of different levels of action, political, scientific and technical; the results fed the debates, and conversely, census categories were also constructed in relation to the political context. The treatment of the insanity statistics of free blacks in 1840, the creation of the mulatto category in 1850 in order to maintain mixed-race individuals inside the population defined as black and in the hope of documenting the degeneracy of the "hybrids", and finally the attempt, starting in 1870 , to define the offspring of immigrants by inherited traits and not just by place of birth, show how political questions of population were also conceived in biological terms. 
PAUL SCHOR 